\title{
KINETIC ENERGY HARVESTING
}

\author{
S.P. Beeby, R.N. Torah and M.J. Tudor \\ School of Electronics and Computer Science, University of Southampton, UK \\ spb@ecs.soton.ac.uk
}

\begin{abstract}
This paper reviews kinetic energy harvesting as a potential localised power supply for wireless applications. Harvesting devices are typically implemented as resonant devices of which the power output depends upon the size of the inertial mass, the frequency and amplitude of the driving vibrations, the maximum available mass displacement and the damping. Three transduction mechanisms are currently primarily employed to convert mechanical into electrical energy: electromagnetic, piezoelectric and electrostatic. Piezoelectric and electrostatic mechanisms are best suited to small size MEMS implementations, but the power output from such devices is at present limited to a few microwatts. An electromagnetic generator implemented with discrete components has produced a power $120 \mu \mathrm{W}$ with the highest recorded efficiency to date of $51 \%$ for a device of this size reported to date. The packaged device is $0.8 \mathrm{~cm}^{3}$ and weighs 1.6 grams. The suitability of the technology in space applications will be determined by the nature of the available kinetic energy and the required level of output power. A radioactively coupled device may present an opportunity where suitable vibrations do not exist.
\end{abstract}

\section{INTRODUCTION}

Wireless systems are becoming ubiquitous and the use of wireless devices offers several advantages over existing, wired methodologies. These include flexibility, ease of implementation and the ability to place sensors in previously inaccessible locations. Furthermore, in the case of wired solutions, the wires (and associated connectors) are often a source of failure, add weight and present a considerable cost. The ability to retrofit systems without having to consider issues such as cabling, offers a significant advantage in applications for areas like condition-based monitoring [1].

At present, many wireless sensor nodes are battery-powered and operate on an extremely economical energy budget since continuous battery replacement is not an option for networks with thousands of physically embedded nodes [2]. The low power characteristics of wireless sensor network components and the design of the system architecture are crucial to the longevity of the sensor nodes. Low power wireless communications protocols (e.g. IEEE 802.15.4 [3] and Zigbee [4]) and the use of intelligence at the sensor node to perform signal processing on the raw sensor data, execute communications protocols and manage the node's power consumption have resulted in very lowpower wireless sensor nodes. These provide the opportunity for alternative types of power source to traditional batteries. Renewable power can be obtained by generating electrical energy from light, thermal and kinetic energy present within the sensor's environment. These sources can be used as either a direct replacement or to augment the battery, thereby increasing the lifetime and capability of the network [5-8] and mitigate the environmental impact caused by issues surrounding the disposal of batteries. 
The subject of this paper is kinetic energy generators, which convert energy in the form of movement present in the application environment into electrical energy [9]. Kinetic energy is typically present in the form of vibrations, random displacements or forces and is typically converted into electrical energy using electromagnetic, piezoelectric or electrostatic mechanisms. Suitable vibrations can be found in numerous applications including common household goods (e.g. fridges, washing machines, microwave ovens), industrial plant equipment, automobiles and aeroplanes and structures such as buildings and bridges [10]. Human-based applications are characterised by low frequency high amplitude displacements [11, 12].

The amount of energy generated by this approach depends fundamentally upon the quantity and form of the kinetic energy available in the application environment and the efficiency of the generator and the power conversion electronics. The following sections will discuss the fundamentals of kinetic energy harvesting and the different transduction mechanisms that may be employed. These mechanisms will then be illustrated by the results from a recently completed EU project 'Vibration Energy Scavenging' (VIBES), the results from which represent the state of the art.

\section{THEORY}

Kinetic energy harvesting requires a transduction mechanism to generate electrical energy from motion and a mechanical system that couples environmental displacements to the transduction mechanism. The design of the mechanical system should maximise the coupling between the kinetic energy source and the transduction mechanism and will depend entirely upon the characteristics of the environmental motion. Vibration energy is best suited to inertial generators with the mechanical component attached to an inertial frame which acts as the fixed reference (see figure 1).

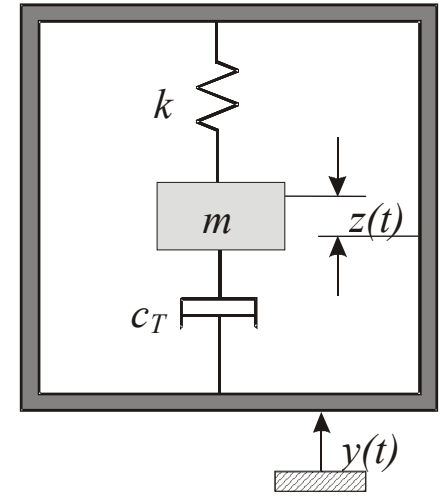

Fig. 1 - Inertial mass with spring stiffness k, mass displacement $\mathrm{z}(\mathrm{t})$, damping coefficient $\mathrm{C}_{\mathrm{T}}$ and input amplitude $y(t)$

The inertial frame transmits the vibrations to a suspended inertial mass producing a relative displacement between them. Such a system will possess a resonant frequency which must be designed to match the characteristic frequency of the application environment. The resonant approach magnifies the environmental vibration amplitude by the quality factor (Q-factor, $Q_{T}$ ) of the resonant system. The Q-factor is limited by damping affects such as unwanted losses and the transduction mechanism used to convert kinetic into electrical energy.

The limitation of the resonant approach is the need to operate at a particular frequency. This limitation can be overcome in part by using a wide bandwidth device, but this approach will reduce the peak power generated. Alternatively, bi-stable structures that flip between states can be employed. This approach will work at any frequency provided the external movement provides sufficient acceleration, but is complicated to achieve in practice and will result in reduced power densities in the majority of applications, depending upon the nature of the excitation $[13,14]$.

The theory of inertial-based generators is well documented [15] and will only be briefly summarized here. Assuming the generator is driven by a harmonic base excitation $y(t)=Y \sin (\omega t)$, it will move out of phase with the mass at resonance resulting in a net displacement, $z(t)$, 
between the mass and the frame. The average power dissipated within the damper (i.e. the power extracted by the transduction mechanism and the power lost through parasitic damping mechanisms) is given by:

$$
P_{a v}=\frac{m \xi_{T} Y^{2}\left(\frac{\omega}{\omega_{n}}\right)^{3} \omega^{3}}{\left[1-\left(\frac{\omega}{\omega_{n}}\right)^{2}\right]^{2}+\left[2 \xi_{T}\left(\frac{\omega}{\omega_{n}}\right)\right]^{2}}
$$

where $\xi_{T}$ is the total damping ratio given by $\xi_{T}=c_{T} / 2 m \omega_{n}$. Since this equation is valid for steady state conditions, $P_{a v}$ is equal to the kinetic energy supplied per second by the application vibrations. Maximum power dissipation within the generator occurs when the device is operated at $\omega_{n}$ and in this case the $P_{a v}$ is given by:

$$
P_{a v}=\frac{m Y^{2} \omega_{n}^{3}}{4 \xi_{T}}
$$

Equation 2 suggests the following rules: (a) power varies linearly with the mass; (b) power increases with the cube of the frequency; and (c) power increases with the square of the base amplitude. Rules (b) and (c) are dependant upon the base excitation i.e. the accelerations present in the application environment. Since the peak acceleration of the base, $A$, is given by $A=\omega^{2} Y$ and damping factor is related the damping ratio by $c_{T}=2 m \omega_{n} \xi_{T}$, equation 2 can also be written in the form:

$P_{a v}=\frac{(m A)^{2}}{2 c_{T}}$

These equations emphasize the need to understand the vibrations present in the intended application when designing an inertial generator. However, one cannot simply choose a particular frequency of operation based upon the power output alone. The inertial mass displacement will be limited to a given finite value, $z_{\max }$, depending upon the size of the generator, its design and material limitations. This is especially relevant in the case of MEMS generators. Furthermore, $z_{\max }$ will be a multiple $Q_{T}$ times larger than $Y$ where $Q_{T}$ is given by equation 4 :

$$
Q_{T}=\frac{\omega_{n} m}{c_{T}}=\frac{1}{2 \xi_{T}}
$$

The relationship between $Q_{T}$ and the electrical and parasitic damping factors is given by equation 5 where $Q_{O C}$ is the open circuit Q-factor, i.e. $1 / 2 \xi_{P}$, and $Q_{E}$ is equal to $1 / 2 \xi_{E}$.

$$
\frac{1}{Q_{T}}=\frac{1}{Q_{O C}}+\frac{1}{Q_{E}}
$$

Taking $z_{\max }$ into consideration, average power can also be expressed as:

$$
P_{a v}=\frac{m \omega_{n}^{3} Y z_{\max }}{2}
$$

Incorporating the parasitic and electrical damping into equation 2 gives the average power delivered to the electrical domain:

$$
P_{\text {avelec }}=\frac{m \xi_{E} Y^{2} \omega_{n}^{3}}{4\left(\xi_{P}+\xi_{E}\right)^{2}}
$$

Maximum power is delivered to electrical domain when $\xi_{E}=\xi_{P}$ i.e. damping arising from the electrical domain should equal mechanical losses. This applies when $z(t)$ is less than or equal to $z_{\max }$. In this case equation 7 simplifies to:

$P_{\text {avelec }}=\frac{m Y^{2} \omega_{n}^{3}}{16 \xi_{P}}$

In many cases and especially for small devices, it is best to maximise the electrical damping and minimise parasitic losses due to the restricted $\mathrm{z}_{\max }$ for $\mathrm{a}$ particular device.

\section{TRANSDUCTION}

The transduction mechanism itself can generate electricity by exploiting the mechanical strain or relative displacement occurring within the system. The strain effect exploits a deformation and typically employs active materials (e.g. piezoelectric) to generate electrical energy from the resulting strain. In the case of relative displacement, either the velocity 
or position can be used. Velocity is typically associated with electromagnetic transduction whist relative position is associated with electrostatic transduction. Each transduction mechanism exhibits different damping characteristics and this should be taken into consideration when modelling the generators.

\section{Piezoelectric Transduction}

The piezoelectric effect arises when certain materials subject to mechanical strain become electrically polarized. Conversely, these materials deform when exposed to an electric field. Piezoelectric materials are widely available in many forms including single crystal (e.g. quartz), piezoceramic (e.g. lead zirconate titanate or PZT), thin film (e.g. sputtered zinc oxide), screen printable thick-films based upon piezoceramic powders [16] and polymeric materials such as Polyvinylidenefluoride (PVDF) [17].

Piezoelectric materials exhibit anisotropic characteristics i.e. the properties of the material differ depending upon the direction of forces and orientation of the polarisation and electrodes. The level of piezoelectric activity of a material is defined by a series of constants used in conjunction with the axes notation. The piezoelectric strain constant, $d$, relates the strain developed to the applied field. Piezoelectric generators that rely on a compressive strain applied perpendicular to the electrodes exploit the $d_{33}$ coefficient of the material. Typically, in the case of piezoelectric films or piezoelectric elements bonded onto substrates, the elements are coupled with a transverse strain parallel to the electrodes and utilise the $d_{31}$ coefficient. Such an arrangement provides mechanical amplification of the applied stresses.

Another important constant determining the generation of electrical power is the electro-mechanical coupling coefficient, $\mathrm{k}$. This describes the efficiency with which the energy is converted by the material between electrical and mechanical domains. It is given by equation 9 where $W_{e}$ and $W_{m}$ are electrical and mechanical energy respectively.

$$
k^{2}=\frac{W_{e}}{W_{m}}
$$

Piezoelectric properties vary with age, stress and temperature. The aging rate of a piezoceramic is dependant on the construction methods and the material type. The changes in the material tend to be logarithmic with time, thus the material properties stabilise with age. The aging process is accelerated by the stress applied to the material and this should be considered in cyclically loaded energy harvesting applications. Soft piezoceramic compositions, such as PZT-5H, are more susceptible to stress induced changes than the harder compositions such as PZT-5A. Maximum temperature of a piezoceramic is limited to the Curie point above which it becomes de-poled. The application of stress can lower this Curie temperature making high stress, high temperature applications challenging. The piezoelectric constants for common materials, soft and hard lead zirconate titanate piezoceramics (PZT-5H and PZT-5A), barium titanate (BaTiO3) and PVDF, are given in Table 1.

Table 1: Coefficients of common piezoelectric materials $[18,19]$

\begin{tabular}{|l|c|c|c|c|}
\hline Property & $\begin{array}{c}\text { PZT- } \\
5 \mathrm{H}\end{array}$ & $\begin{array}{c}\text { PZT- } \\
5 \mathrm{~A}\end{array}$ & BaTiO $_{3}$ & PVDF \\
\hline $\begin{array}{l}\mathrm{d}_{33}\left(10^{-12}\right. \\
\mathrm{C} / \mathrm{N})\end{array}$ & 593 & 374 & 149 & -33 \\
\hline $\begin{array}{l}\mathrm{d}_{31}\left(10^{-12}\right. \\
\mathrm{C} / \mathrm{N})\end{array}$ & -274 & -171 & 78 & 23 \\
\hline $\mathrm{k}_{33}$ & 0.75 & 0.71 & 0.48 & 0.15 \\
\hline $\mathrm{k}_{31}$ & 0.39 & 0.31 & 0.21 & 0.12 \\
\hline $\begin{array}{l}\text { Curie Temp } \\
(\mathrm{C})\end{array}$ & 195 & 365 & 120 & 110 \\
\hline
\end{tabular}

\section{Electromagnetic Transduction}

Electromagnetic induction is the generation of electric current in a conductor located within a magnetic field. The conductor typically takes the form of a coil and the electricity is generated by either the relative movement of the magnet and coil, or because of changes in the magnetic field. In the former case, the amount of electricity generated depends upon the strength of the magnetic field, the velocity of the relative motion and the number of turns of the coil. 
The damping coefficient arising from electromagnetic transduction $c_{e}$ can be estimated from equation 10 [20].

$$
c_{e}=\frac{(N l B)^{2}}{R_{\text {load }}+R_{\text {coil }}+j \omega L_{\text {coil }}}
$$

where $N$ is the number of turns in the generator coil, $l$ is the side length of the coil (assumed square), and $B$ is the flux density to which it is subjected and $R_{\text {load }}$, $R_{\text {coil }}$, and $L_{\text {coil }}$ are the load resistance, coil resistance and coil inductance respectively. Equation 10 shows that $R_{\text {load }}$ can be used to adjust $c_{e}$ to match $c_{p}$ and therefore maximise power, although this must be done with the coil parameters in mind. It can be shown that the maximum average power delivered to the load can be found from equation 11 [15].

$$
P_{\text {eload } \max }=\frac{m A^{2}}{16 \zeta_{p} \omega_{n}}\left(1-\frac{R_{\text {coil }}}{R_{\text {load }}}\right)
$$

\section{Electrostatic Transduction}

Electrostatic transduction exploits the relative movement between two dielectrically isolated electrodes (capacitor). These plates are charged by periodic connection to a voltage source or by the use of electrets. The energy stored in a capacitor, with plate charge $Q$ and potential difference $\mathrm{V}$, is given by:

$\mathrm{E}=0.5 \mathrm{QV}=0.5 \mathrm{CV}^{2}=0.5 \mathrm{Q}^{2} / \mathrm{C}$

If the charge on the plates is held constant the perpendicular force between the plates is given by:

$$
\mathrm{F}=0.5 \mathrm{Q} 2 \mathrm{~d} / \varepsilon \mathrm{A}
$$

If the voltage between the plates is held constant the perpendicular force between the plates is given by:

$$
\mathrm{F}=0.5 \varepsilon \mathrm{AV}^{2} / \mathrm{d}^{2}
$$

The work done against the electrostatic force between the plates provides the harvested energy.

In MEMS the separation between the two plates is typically very small (nm to $\mu \mathrm{m}$ range). Electrostatic generators can be classified into three types [21]:
1) In plane overlap varying (Fig. 2)

2) In plane gap closing (Fig. 3)

3) Out of plane gap closing (Fig. 4)

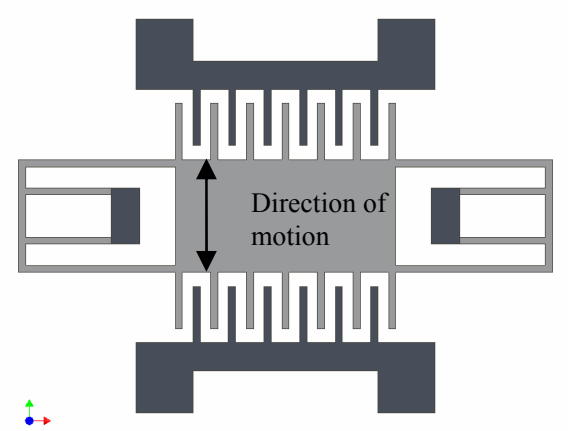

Fig 2: In plane overlap varying

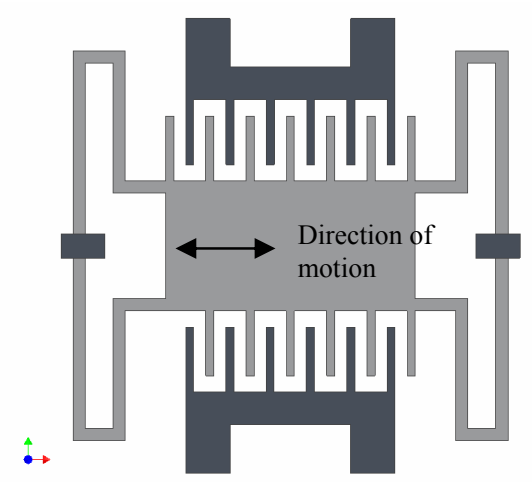

Fig 3: In plane gap closing

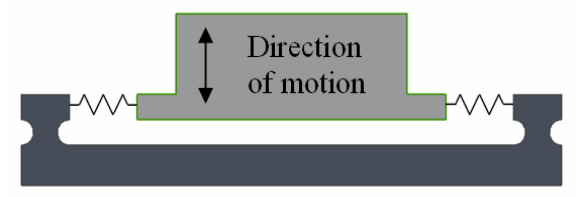

Fig 4: Out of plane gap closing

Note both in plane configurations create two variable capacitors with the capacitances $180^{\circ}$ out of phase. The three approaches can be operated either in charge constrained or voltage constrained cycles. In general the voltage constrained offers more energy than the charge constrained approach. However, by incorporating a capacitor in parallel with the energy harvesting capacitor, the energy from the charge constrained system can approach that of the voltage constrained system as the parallel capacitance approaches infinity. This parallel capacitor effectively constrains the voltage on the energy harvesting capacitor [22]. Maximum power generation occurs for very small dielectric gaps in which case 
the source impedance can be very high resulting in poor power delivery.

Table 2 provides the electrostatic force variation for the three configurations where $x$ is the displacement of the inertial mass [23]. For a high damping configuration the electrostatic damping force has to be counter balanced almost entirely by the mechanical spring force.

Table 2: Electrostatic force variation for the three configurations

\begin{tabular}{|l|l|l|}
\hline Structure & $\begin{array}{l}\text { Charge } \\
\text { constrained }\end{array}$ & $\begin{array}{l}\text { Voltage } \\
\text { constrained }\end{array}$ \\
\hline $\begin{array}{l}\text { In plane overlap } \\
\text { varying }\end{array}$ & $\mathrm{F}_{\mathrm{e}} \sim 1 / \mathrm{x}^{2}$ & $\mathrm{~F}_{\mathrm{e}}$ constant \\
\hline $\begin{array}{l}\text { In plane gap } \\
\text { closing }\end{array}$ & $\mathrm{F}_{\mathrm{e}} \sim \mathrm{x}$ & $\mathrm{F}_{\mathrm{e}} \sim 1 / \mathrm{x}^{2}$ \\
\hline $\begin{array}{l}\text { Out of plane } \\
\text { gap closing }\end{array}$ & $\mathrm{F}_{\mathrm{e}}$ constant & $\mathrm{F}_{\mathrm{e}} \sim 1 / \mathrm{x}$ \\
\hline
\end{tabular}

\section{STATE OF THE ART}

The state of the art in electromagnetic and piezoelectric vibration power generators has been achieved in the VIBES project.

\section{Electromagnetic Generator}

The micro electromagnetic generator was designed to operate at $50 \mathrm{~Hz}$ targeting electrical equipment driven by mains electricity [24]. The generator design is based upon a cantilever beam spring element and uses miniature discrete components fabricated using a variety of conventional manufacturing processes. This enables the generator to exploit the advantages of bulk magnetic material properties and large coil winding density thereby demonstrating very useable levels of power from a compact design. The design uses four high energy density sintered rare earth Neodymium Iron Boron (NdFeB) magnets manually bonded to the top and bottom surfaces of a cantilever beam. The magnetic poles are aligned as shown in figure 3 . The magnetic circuit is completed by zinc coated mild steel keepers which couple the flux between top and bottom magnets. This arrangement produces a concentrated flux gradient through the stationary coil as the magnets vibrate.

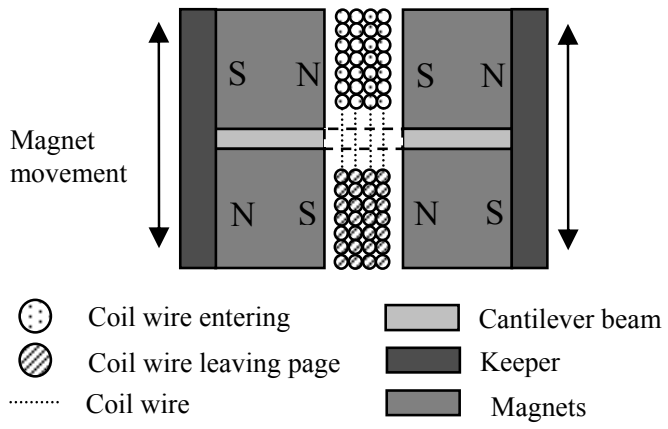

Fig 3. Cross section through the four magnet arrangement.

Additional mass is added to the generator in the form of wire eroded tungsten alloy blocks attached to the free end of the cantilever beam. The tungsten alloy has a density of $18.1 \mathrm{~g} / \mathrm{cm}^{3}$ providing a compact inertial mass. Adjustment of the generator's resonant frequency can be achieved by altering the cantilever beam length. ANSYS modal analysis predicted an adjustment of $1.3 \mathrm{~mm}$ would produce a frequency range of $44-60 \mathrm{~Hz}$. Therefore, a slot was etched into the beam at the clamping point to allow for manual adjustment of the beam length Additionally, the coil has outer and inner diameters of $2.8 \mathrm{~mm}$ and $0.5 \mathrm{~mm}$ respectively. It is wound with $12 \mu \mathrm{m}$ diameter copper wire achieving 2800 turns and a coil resistance of $2.3 \mathrm{k} \Omega$. The cantilever beam assembly was clamped onto the base using an M1 sized nut and bolt and a square washer. The square washer gives a straight clamped edge perpendicular to beam length. The coil was manually bonded to a semi-circular recess machined in the base. A drawing of the assembled generator is shown in figure 4. With the aid of alignment jigs, a tolerance of better than $0.1 \mathrm{~mm}$ can be achieved with the manual assembly of the components. The practical volume, i.e. including the swept volume of the beam, is approximately $0.15 \mathrm{~cm}^{3}$, but this increases to $0.8 \mathrm{~cm}^{3}$ when packaged with a protective lid and weighs $1.6 \mathrm{~g}$. 


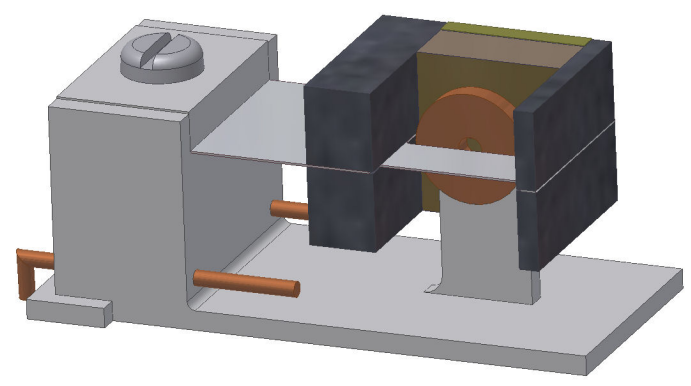

Fig 4. Micro cantilever generator.

The generator performance was evaluated using an accelerometer feedback controlled shaker unit with a programmable resistive load. The tests involve sweeping through a range of frequencies with varying load resistances from 100 to $9 \mathrm{M} \Omega$. The generator was subjected to a vibration acceleration of 0.6 $\mathrm{ms}^{-2}$ throughout the tests. The optimum power output of $58 \mu \mathrm{W}$ and a voltage of $1.12 \mathrm{~V}$ was achieved with a $15 \mathrm{k} \Omega$ load resistance. Connection to a voltage multiplier circuit and supercapacitor for energy storage results in a reduction in peak power to $37 \mu \mathrm{W}$ but the useable bandwidth increases to over $1 \mathrm{~Hz}$ (see figure 5). This is due to the in increased damping arising from the improved coupling provided by a capacitive load [25].

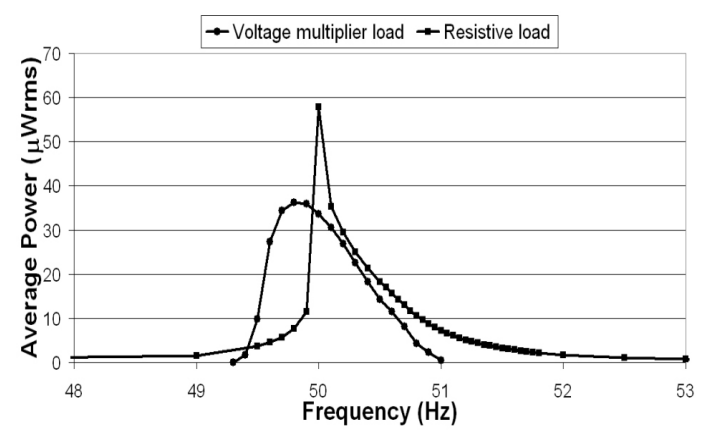

Fig. 5: Maximum generated power into a resistive load of $15 \mathrm{k} \Omega$ and into the voltage multiplier circuit, both at $0.6 \mathrm{~ms}^{-2}$ vibration.

This increased damping means the generator can be driven up to an acceleration level of $1.7 \mathrm{~ms}^{-2}$ before reaching $z_{\max }$ at which point the power output is $120 \mu \mathrm{Wrms}$. This device converts $51 \%$ of the energy from the mechanical domain to the electric domain.

\section{Piezoelectric Generator}

The piezoelectric generators developed in the VIBES projects are MEMS fabricated using silicon micromachining and thin film deposition techniques for the piezoelectric material. These generators have been designed to match the higher frequency vibrations associated with a machine tool application $(>800 \mathrm{~Hz})$. The design is based upon a cantilever structure with a silicon inertial mass as shown in figure 6 .

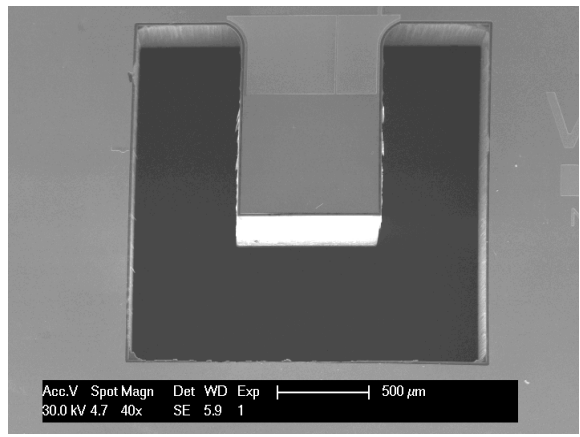

Fig 6: SEM photo of piezoelectric device.

Two types of piezoelectric materials have been investigated: PZT and AIN. The AIN devices produced a maximum output power of $2 \mu \mathrm{W}$ with voltage amplitude of about $1.6 \mathrm{~V}$ at $39 \mathrm{~m} / \mathrm{s}^{2}$ acceleration. The PZT devices have been fabricated with three different electrode arrangements and the maximum power of $1.4 \mu \mathrm{W}$ at 19.6 $\mathrm{m} / \mathrm{s}^{2}$ acceleration amplitude was obtained with an IDT electrode arrangement.

Despite the low power output, the piezoelectric generator was combined with discrete electronic components and used to power a temperature sensing wireless system. The system was duty cycled to wake up from sleep every 30 seconds, measure voltage on capacitor and if the voltage was higher than $2.1 \mathrm{~V}$ take a temperature sensor reading $(200 \mu \mathrm{s})$ and transmit 5 Bytes. This system was able to take measurements every 2 minutes.

\section{SPACE APPLICATIONS}

To the authors' knowledge, vibration energy harvesting has yet to be employed in space applications. Its potential is dependant upon the availability and nature of kinetic energy within potential 
application environments. Another consideration is the size of the generators. It is desirable for devices deployed in space to be as small and low mass as possible. However, in typical inertial vibration powered generators, the power output reduces linearly with mass, and therefore smaller devices produce less power output. Furthermore, the efficiency of microscale inertial generators is relatively poor at present. Electrostatic and piezoelectric devices are best suited to MEMS scale producing a few microwatts of power (depending upon excitation). Electromagnetics do not scale down well in size, which coupled with the reduced mass, leads to very low levels of power output (e.g. $<1 \mu \mathrm{W})$. A beneficial aspect of operating in space will be the low pressure environment which removes gas damping effects.

One potential method for implementing this technology in space applications where no suitable vibrations are present is to provide an alternative method for actuating the generator. This has been demonstrated in the case of a piezoelectric cantilever beam which was coupled to radioactive source $[26,27]$. The principle uses the radiated $\beta$ particles to electrostatically charge a conductive plate on the underside of the cantilever. As the electrostatic field builds, the beam is attracted to the source until contact is made and the field dissipated. At this point, the beam is released to vibrate at its natural frequency and the kinetic energy harvested from the piezoelectric film. A schematic of the device is shown in figure 7. Different material combinations, device geometries and radioisotopes can alter the output and characteristics of the generator. For example, a $1 \mathrm{~cm}$ square 0.5 millicurie thin film ${ }^{63} \mathrm{Ni}$ source with a half life of 100.2 years coupled to a $15 \mathrm{~mm}$ long, 2 mm wide silicon cantilever produced a peak power of $16 \mu \mathrm{W}$ with a reciprocation period of 115 minutes [28]. This presents a novel and repeatable method for exciting the cantilevers vibrations and will work for very long time periods certainly more than alternative local power supplies such as batteries. The power output is very periodic and, when averaged out over a given time period, very low $(<1 \mathrm{nW})$.

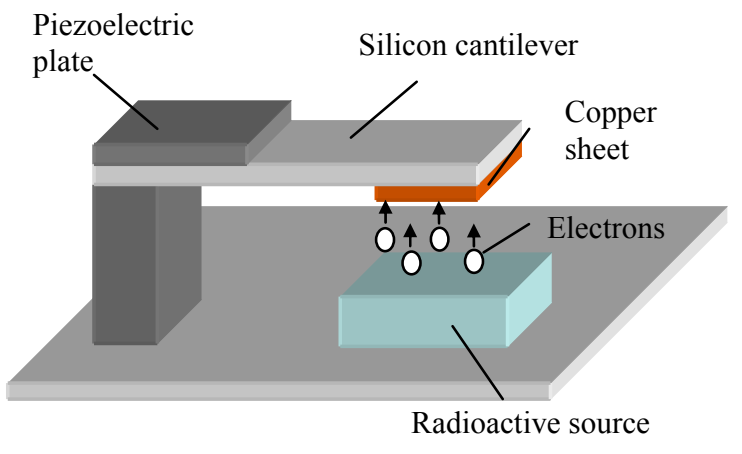

Fig 7 - Radiation driven piezoelectric generator

\section{CONCLUSIONS}

This paper has reviewed vibration energy harvesting used to date to provide a localised power supply for wireless applications. The three transduction mechanisms employed to date to convert mechanical energy into electrical were presented along the characterising equations that highlight the important design parameters. Generator design and performance is inexorably linked to nature of the environmental excitation energy and successful implementation requires accurate knowledge of the application. Its suitability for space applications will be determined by the nature of the available kinetic energy. The radioactively coupled device may present an opportunity where suitable vibrations do not exist.

\section{ACKNOWLEDGMENTS}

The authors would like to acknowledge funding for the VIBES project from the European Union, and the VIBES consortium, in particular our colleagues at the Tyndall National Research Centre, TIMA and EPFL.

\section{REFERENCES}

[1] Bult K., et al. 'A Distributed, Wireless MEMS Technology for Condition Based Maintenance' Proc 1996 Integrated Monitoring, Diagnostics and Failure Prevention Conference, Society of Machine Failure Protection Technology 
(MPFT) (Mobile, Alabama, USA) pp 373380

[2] Raghunathan V., Schurgers C., Park S. and Skrivastava M.B., 'Energy-Aware Wireless Microsensor Networks' IEEE Signal Processing magazine 19, 2002, pp. 40-50

[3] IEEE 802.15.4 Standard, 2003 (New York, The Institute of Electrical and Electronics Engineers, Inc)

[4] Frank R. 'Move Over, Bluetooth; ZigBee is here' Design News 42004 (http://www.designnews.com/article/CA38 7448.html).

[5] Koeneman P.B., Busche-Vishniac I.J. and Wood K.L. 'Feasibility of micro power supplies for MEMS' IEEE J. Microelectomechanical Systems 6, 1997, pp. 355- 362

[6] Görge G., Kirstein M. and Erbel R. 'Microgenerators for energy autarkic pacemakers and defibrillators: fact or fiction' Herz, 26, 2001, pp. 64-68

[7]. Amirtharajah R. and Chandrakasan A.P. 'Self-Powered Signal Processing Using Vibration-Based Power Generation' IEEE J of Solid State Circuits 33, 1998, pp. 687-695

[8] Jacobson S.A. and Epstein A.H. 'An informal survey of power MEMS' Proc. The Int. Symp. on Micro-Mechanical Engineering ISMME (Japan) 2003, pp. K18

[9] Beeby S.P., Tudor M.J. and White N.M. 'Energy harvesting vibration sources for microsystems applications' J. Meas. Sci. Technol. 17, 2006, pp. R175-R195

[10] Roundy S., Wright P.K. and Rabaye J. 'A study of low level vibrations as a power source for wireless sensor nodes' Computer Communications 26, 2003, pp.1131-1144.

[11] Starner T. and Paradiso J.A. 'Human Generated Power for Mobile Electronics' in Low Power Electronics Design, ed. C Piguet (Boca Raton, Florida: CRC Press LLC, 2004)

[12] von Büren T., Lukowicz P. and Tröster G. 'Kinetic Energy Powered Computing - an Experimental Feasibility Study' Proc. $7^{\text {th }}$ IEEE Int. Symposium on Wearable Computers ISWC '03(White Plains, NY) 2003, pp. 22-24

[13] Mitcheson P., Stark B., Miao P., Yeatman E., Holmes A. and Green T.,
'Analysis and optimisation of MEMS onchip power supply for self powering of slow moving sensors', Eurosensors 03, 17th European conference on sensors and actuators, Guimaraes, Portugal, 21-24 September 2003, pp. 48 - 51

[14] Elliot, S.J., Saba R. And Baumann O.N. 'Vibraion power harvesting from head motion', Accepted 7th European Conference of Structural Dynamics, Eurodyn 2008, 7-9th July Southampton, UK

[15] Stephen N.G. 'On energy harvesting from ambient vibration' Journal of Sound and Vibration 293, 2006, pp. 409-425

[16] Baudry H. Screen-printing piezoelectric devices Proc. $6^{\text {th }}$ European Microelectronics Conference (London, UK) 1987 pp. 456-463

[17] Lovinger A. J Ferroelectric Polymers, Science 220, 1983, pp. 1115-1121.

[18] Piezoelectric Ceramics Data Book for Designers, Morgans Electroceramics

[19] Gonzalez J.L., Rubio A. and Moll F. 'A prospect of the of piezoelectric effect to supply power to wearable electronic devices' Proc. $4^{\text {th }}$ Int. Conf. on Materials Engineering for Resources (Akita, Japan) 2001pp. 202-207

[20] El-Hami M. Et al, 'Design and fabrication of a new vibration-based electromechanical power generator' Sensors and Actuators A92, 2001 pp. 335342

[21] Roundy S., Wright P. and Pister K. 'Micro-electrostatic vibration-to-electricity converters' Proceeding of IMECE, 2002, pp. 1-10.

[22] Meninger S. 'A low power controller for a MEMS based energy converter', MSc Thesis, Massachusetts Institute of Technology, 1999

[23] Despesse G. et al, 'Fabrication and characterisation of high damping electrostatic micro devices for vibration energy scavenging' Proc. Design, Test, Integration and Packaging of MEMS and MOEMS, 2005, pp. 386-390.

[24] Beeby S. et al, 'Micro electromagnetic generator for vibration energy harvesting' J. of Micromech. Microeng. 17, 2007, pp. 1257-1265

[25] Torah R. N. et al, 'Energy aware wireless microsystem powered by vibration energy harvesting', Proc 
ACT Workshop on Innovative Concepts. ESA-ESTEC 28-29 January 2008.

PowerMEMS 2007，28-29 ${ }^{\text {th }}$ November 2007, Freiburg, Germany, pp. 323-326.

[26] Li H., Lal A., Blanchard J. and Henderson D 'Self-reciprocating radioisotope-powered cantilever' J. App. Phys. 92, 2002, pp. 1122-1127
[27] Lal A. and Blanchard J. 'The daintiest dynamics' IEEE Spectrum 41 2004, pp. 36-41

[28] Duggirala R., et al 'Radioisotope micropower generator for CMOS selfpowered sensor microsystems' Proc. PowerMEMS 2004 (Kyoto, Japan) pp. 133-136 